\title{
A Review on advancements in Nano memory devices
}

\author{
${ }^{1}$ V G Hamsaveni, ${ }^{2}$ Kiran Agarwal \\ \{1ghamsaveni@gmail.com, ${ }^{2}$ drkirangupta15@gmail.com $\}$
}

${ }^{1}$ Ph.D. Scholar, Department of Electronics and Communication DSCE, Visvesvaraya Technological University, Bangalore, MIEEE Professor Department of Electronics and Communication DSCE, Visvesvaraya Technological University, Bangalore ${ }^{2}$

\begin{abstract}
The In this era computing is one of the most prominent field and in the past 20 years the growth in the computers has improved with the combined growth in the field of electronics. Memory devices plays a major role in the most of the devices such as memory storage device and memory processing device which enables the fast computing and vast storage capacity. This paper reviews the history of the storage systems. Beginning with the mechanical calculators, magnetic tapes, magnetic disks, floppy disk, optical devices, Hard disk drive, solid state drive, SRAM, DRAM, graphene based memory with emerging technologies like Artificial intelligence, Bigdata, cloud computing, IoT, and blockchain, to the increasing demand in the in the computing high performing devices are a must and this includes and brief approach of the concept oriented research which involves with the Nano dot based memory devices which can enhance the future scope in memory devices, networking and cloud storage. This literature survey is an review for our work which we would like to present in our future projects and it gives a brief outcome of the research based on the synthesis of gold ( $\mathrm{Au})$ Nanodots $\alpha-\mathrm{Fe} 2 \mathrm{O} 3$.
\end{abstract}

Keywords: Nanomemory, Nanodots, Storage Device Evolution.

\section{Introduction}

In Today's world data is the most important thing and we are using lot of storage devices to store our day to day memory. The memory storage devices have improved when compared to the last decade it has shown a drastic improvement corresponding with Moore's law. The rapid development in the memory has shown an impact in the storage media and the large disk drives are turned into Nano devices that has enabled and shown new possibilities in the field of research which has an active possibility in the field of Nano memory.

In main goal in this article is we are discussing about the brief literature of existing technologies like the storage devices, $2 \mathrm{~d}$ materials, rram, stt-mram, hdd, ssd and our contribution in making of the device which can improve the efficiency in terms of size and compatibility. It is observed that the research in the Nano materials field is restricted to the industry. Here we are using an process to improve data storage density, better performance and decreasing the size of the device. we use the synthesis of gold ( $\mathrm{Au}$ ) with Nanodots $\alpha$ Fe2O3 using sol-gel method. 


\section{Existing Technologies}

1. STORAGE MEDIA (INCEPTION - PRESENT): Earliest computers used papers for the memory storage, after the pulp making process. In 1825 Paper was an primary material in the storage device. Later in 1880 s it was replaced by the punch cards in the mechanical calculators that was invented to be used as a statistical tabulation. It was used to tabulate the census at that time based on the tabulations IBM was formed. Punching cards played a major role in mid 1980s. The usage of the materials has gradually increased year by year with opening a billion-dollar market. Introducing the magnetic tape which was manufactured using the ferric oxide powder coated this was used for sound and media. It was later used in manufacturing the phonograph records. Magnetic disks started revolutionizing it begun with IBM disk file developed which was controlled using servo motors it was built using the metal platters coated with magnetic materials and it stored around $3.75 \mathrm{MB}$ of information and transfer rate of 8800 characters per second. It was reduced to smaller sized disks according to the market necessity. It has transformed from 14, 8,5 inch platters. This has opened an wide market opportunity for the compact disks (CDs) and Digital versatile disc (DVDs). It had an option such as write once and read many (WORM) that had no option but to buy and store many disks. And the rewritable disks were found as an alternate to this model which disrupted the market with the $4.7 \mathrm{~GB}, 8.5 \mathrm{~GB}, 9.4 \mathrm{~GB}, 17.8 \mathrm{~GB}$ which is till date an alternate to the cost efficiency mode of memory transfer.

SCM - Storage Class Memory is the present and most efficient way of transferring the data from one system to other systems. Flash memory is the present and ongoing which is a sort of EEPROM electrically erasable programmable read only memory. Here the memory of the controller is capable of program or erase the information by putting or removing an electron to or from solid state cell made of a floating gate and read information by measuring voltage of the floating gate. This design particularly improvised the quality of the storage devices in the field of computers. Till date it is the dominant technology for the memory devices. Active research is being conducted in order to improvise the performance, reliability, and recording speed. Storage Networking is the latest and it is facing the new heights in terms performance, speed and reliability through the cloud computing storage technologies in the IT industry. Storage Area Network (SAN) and Network Attached Storage (NAS) are making an great access in the private networks where the data can be stored at home and accessed anywhere across the globe.

2. 2D MATERIALS CHARGE TRANSFER: 2D Materials has been a hot research topic from 2004. It is said that 2D materials has great diversity in bandgap, properties and this provided the platform for the electronic devices with high mechanical strength and allows materials without dangling bonds and it guarantees excellent electronic characteristics. Based on the Van der Walls forces it has a novel opportunity in the 2D materials and in the nanoscale devices. It has an potential solution to improve the memory performance and memory units. The fabrication done using carbon allotropes with a honey comb structure is used here in order to provide mechanical strength and high mobility. Carbon exhibits semi- metal behavior and now is a common parts of memory cells like electrodes or floating cells. Hexagonal boron nitride has a large bandgap and it performs as a insulator with ultra flat and zero defect interface. These honey comb structures can be manufactured in two ways dry transfer and wet transfer. In the first case the material is placed on the target substrate by the mechanical or Chemical Vapor Deposition (CVD) Technique. Then the bottom material is fixed by using the 
specific polymer. The deionized water is used in the polymer dissolution by this method the transfer is achieved.

3. RRAM - CELLS \& SYSTEM: In accordance with the fast program read speed and low power consumption and larger scalability the Resistive Random Access Memory is said to be an efficient option in the performance of the storage systems. It has attractive advantages to make a system faster in boosting the performance of the system which gives user rapid speed, performance and it is used for the high-density storage and accessible application in the modern computers. It is very complicated when compared with the previous generation memory it involves in accordance with electrode materials, switching materials, operation mode and temperature. It operates in the high resistance state to low resisting state determined by the SET status. It has two states unipolar and bipolar state when the SET and RESET are in same voltage this is said to be unipolar if they are in different voltage it is bipolar. If both are not applicable and if it is in the unipolar stage it is known as non-polar state. The $\mathrm{Ag}, \mathrm{Cu}, \mathrm{Ni}$, $\mathrm{Pt}, \mathrm{Pd}, \mathrm{Au}$ following electrodes can be used for the performance in the electrochemical layer. The switching layer is of the ionic conductor is of oxidized metal ions. Under the electric field it transfers from active electrode to inert electrode with the influence of the chemical and electric potential field. Primary active electrodes or nano particles in the switching layer and the oxygen ions in the cells are composed of oxygen vacant which can extend from bottom to top electrode or top to bottom electrode based on and the migration towards bottom electode depending on the electrochemical oxidation of oxygen. These thermal effects are unavoidable because of the high electric field in the device operation. Phase-change memory (PRAM) is widely considered memory in the various devices which has high performance. These are used for the high density storage materials used in here CMOS compatibility is the main criteria which refers with the cost- effective and high storage and the device lifespan with performance. In the manufacturing Tantalum oxide (TaOx) and Hafnium oxide (HFOx) are most commonly used in materials. These have a potential to form a multi dimensional memory cell structure. Both the materials has given an positive outcome in the research conducted which could be manufactured within 40nm technology and this has shown positive performance including the operational characteristic outcome. In this paper we would like propose $9 \mathrm{~nm}$ technology oriented with the fundamental mechanism which can be a result oriented outcome.

4. STT-MRAM - CELLS \& SYSTEM: Spin transfer torque magneto-resistive randomaccess memory (STT-MRAM) has been developed 10 years ago which is based on the electrical approach through the magnetization which enables the MRAM with high density storage capability this opened the new opportunities in the very-large scale integrated circuits (VLSI). In the past few years this technology has been developed rapidly with improvements in the increasing storage and performance characteristics. It consists of magnetic tunnel junction diode and a select transistor. the tunnel diode is used to store the information when it is in parallel configuration between two ferromagnetic layers which responds to a low resistance and the series configuration refers to the high resistance state which is controlled selecting the transistor. the magnetization here is obtained by switching the ferromagnetic layer by a write current that is controlled by the select transistor. With the incubation time delay of 1 to $9 \mathrm{~ns}$ it always exists with the spin transfer torque by considering with the thermal effects the incubation time is proportional and inversely proportional to the damping cost of the layer the switching time must be lesser than the switching current. The magneto tunnel junction has a magnet tunnel resistance of $200 \%$ in 
2005 with usage of tunnel resistance and then it has increased over $600 \%$ with usage of $\mathrm{MgO}$ in 2010. The MRAM has a wide range of practical applications which has performance characteristics which includes thermal stability, critical switching, switching speed and transmission which has been observed in the Figure 1 Transmission Electron Spectroscopy (TEM) image of $\mathrm{MgO}$ with STT-MRAM. This process has been widely used in the intel memory applications and the process has a good retention rate. The performance characteristics are shown in the Figure 2, The schematic sketch of single memory cell of STTMRAM is in Figure 3. Usually STT-MRAM is known for its high performance characteristics and its volatile memory. The size and power required is simpler when compared with other technologies.

5. HARD DISK DRIVE (HDD) : This invention has given an tremendous changes in the storage devices where the storage space and it shown a real density in the performance and higher yield capacity in the devices which increased the storage space and it enabled desktop and laptop storage. It has unique storage capability which the previous generations lacked. It has given a tremendous changes in the storage system revolution. It usually consists of platter, spindle, actuator arm, axis and head, ide connector, jumper block and power connector. It usually starts with the platter a circular metallic disc which consists of one or more the platter is made of aluminum and coated with a magnetic later which allows the drive to store bits of memory consistently. The speed is around 200rpm that can go up to 15500 revolutions varying with the size and capacity of the device. The disk is then connected to the disk head with the actuator arm and axis. Magnetic disk drives has undergone changes which impacts in the todays size and capacity of the device. The invention of the higher storage has made the memory prices down with time it has drastically fallen and the performance has increased which todays servers use to store, write, and retrieve data. The HDDs has undergone vast changes with the significant capacity and it has become inexpensive the architecture has changed widely with the time. It is parallely used with the DRAM. To store the data in the system whereas the DRAM acts as a cache memory. These devices can be used to increase the performance and alternate in the portable drives, secondary memory device using caddy.etc..

6. SOLID STATE DEVICE (SSD): It operates on the NAND flash memory technique. The present generation the HDDs are being replaced by the SSDs as their speed is high. It uses IC assemblies to store the data there are few protocols such as Serial Advanced Technology Attachment (SATA) and Serial Attached Small Computer System Interface (SAS) which is comparatively costlier then the HDDs. These are used as a memory in the much smaller laptop computers where it can speedup the computers performance by $95 \%$. It offers less storage and the secondary drive which can be used for the storing data. The SSDs are not cheap they are 2x more expensive than the HDDs. Its architecture is based on DRAM volatile memory which is based on NAND flash. The controller which is based on the embedded processor which controls flash chip it has higher latency and the cache acts as the memory of the previous user data and when powered off it shows exactly where we have stopped the work on restart. Internal RAM in the SSD is operated at high capacity and performance characteristics.

7. CLOUD STORAGE: The cloud industry is emerging with the several vendors with the storage service providers and they have owned data centers with the increasing network technologies from the customer point of view by a web application whole data can be uploaded or downloaded from the assigned data server with end to end encryption from the vendor. Where the storage management is often served as free initially and after certain limit 
the subscription-based payments usually happen in this space. The top speed data with a huge bandwidth is provided with business overview.

\section{Exisiting Methodologies}

1. 3D STORAGE CLASS MEMORY: In this process $\mathrm{Al} 2 \mathrm{O} 3 / \mathrm{TiO} 2$ a hybrid device with memory which is low off current $<1 \mathrm{nA}$, low reset current $<1 \mathrm{nA}, \&$ high on/off ratio $>104$ with the outstanding observations in the read/write margins and ultra-low consumption are confirmed through array simulations.

Fabrication: A Mixture of Tio2 and Al203 of (2-6 nm) is taken and are sequentially deposited in the atomic layer deposition system and the Pt electrode which acts as a buffer layer by using sputtering technique from the Ag target and Te target.

Working: The operation principle of the hybrid memory device which initially exhibits a high threshold voltage because of the CF which is not formed in the memory layer. When

+ Ve bias is applied it shows low threshold voltage is observed. The memory is obtained by read operation in low and high state. Ti buffer is introduced in order to improve the switching variability and $\mathrm{Al} 2 \mathrm{O} 3$ layer with a higher operating voltage than the selector device is expected to obtain stable outcome.

Outcome: ultra thin dual oxide memory device using atomic layer deposition is done and optimized with low off current and high on/off ratio and it has a possibility in manufacture of 3D memory applications.

2. SIMULATION OF RRAM CIRCUITS: Two memory designs of RRAMs with conductive filaments are considered and the ohmic resistance, temperature, signals, voltage ramps \& evolution are studied.

Observation: It has fast write and low voltage non volatile memories are main players in the semiconductors which are alternatives to flash cells. It has a scaling potential $(<10 \mathrm{~nm})$ with low program, fast switching (<10ns), fast retention ( $>10$ years), it supports CMOS processes. In this simulation of RRAM circuit it is reported that the device temperature, simulation time, shape and tunneling currents are efficient. It consists of a following options which includes SET and RESET which acts as bridge. The simulation results for I-V characteristics \& signals are performed.

Simulation: The device and the conductive filament where the top and bottom electrodes which is linked with the electrochemical metallization cells. Each parameter from the I-V curves for the oxide and the thermal conductivity and heat are observed. The step response

in this model is responsive that it is exponential and considered to tunneling current model with max resistance of $(\sim 250 \mathrm{p} \Omega)$

Outcome: Two models of RRAMs are analyzed in the simulation with the LT Spice Tool and it is observed ohmic resistance is linked to the conductive filament which gives an efficient outcome with the cylindrical filaments.

3. NANO FLOATING GATE MEMORY DEVICES: It is a kind of flash memory devices that uses Nano crystals as charge blocking element. That uses areas which are not extensively explored. This has a capability and application that can be organic, printed and flexible with advantages over other memory devices.

Operation: it is based upon the Metal oxide semiconductor transistor devices. In which the contrast, volatility, which have a constant charge it has a floating gate where the electrons can be stored. Electrons are deposited on tunneling oxide and the trap levels, trap sites can be 
controlled by the Vth where it can be used for the erasing operations. Programmed state " 0 " and erased state "1". In advanced flash memory technology it is capable of storing more than 1 bit in a memory cell so the memory stored can be doubled.

Outcome: In Nano floating gate memory it is observed that it has good properties such as particle size, density and distribution. It can be used in high density memory applications. They can also be used in the organic, printed and transparent materials. The programmable memory performance is excellent so it has a future scope in the manufacturing of the memory devices.

4. COAL BASED GRAPHENE STORAGE: Nano particles on a nickel substrate us used using electro deposition technique where the lithium performance of the composites using lithium ion battery is studied.

Synthesis: Graphene is extracted from the coal dust is pulverized and with nitric acid it is undergone a treatment process. Further oil bath is done at $140{ }^{\circ} \mathrm{C}$ for $24 \mathrm{H}$. it is cooled and the nitrate is removed then the graphene structure is obtained.

Outcome: Graphene memory-based synthesis is performed using the vapor deposition and the acid oxidation using the anthracite powder. the electrodeposition method by adjusting the electrolyte solvent ratio it is prepared by two-step electrodeposition with the electrochemical test. It has shown an ideal rate performance. lithium storage performance and it exhibits the bridge effect that improves the porous spaces and efficiency.

5. HIGHLY REPEATABLE SWITCHING CHARECTERISTICS OF MEMORY DEVICE: In this paper Au/TiO2/Ti memory device is discussed. Comparing with the switching charecteristics and the thickness of the material. The application of device is usually used for RAM which has an extremely repeatable SET and RESET the multilevel switching is explained in different models.

Observation: high purity titanium is ultrasonically cleaned for 15 mins using de-ionized water. it is oxidized using Ti using a precisely temperature-controlled furnace. Ti foil is glued to a glass platform and it is formed into a structure with electrical characterization process. In high temperature it is thermally oxidized. The dispersion is observed using XRD and the $\mathrm{TiO} 2$ is found to be $\sim 450 \mathrm{~nm}$ which is carried out using a morphology instrument. The I-V charecteristics are measured using the semiconductor characteristic device which is used in the system sweeping.

Outcome: The characteristics are highly repeatable, multilevel of an $\mathrm{Au} / \mathrm{TiO} / \mathrm{Ti}$ are thermally timed and used in the device which the study is of SET " 1 or 2" and RESET " 0 ". The fabrication possibilities in RRAM with dimensions of $2 * 2 \mathrm{~mm}$ is possible. With the higher operating temperature upto $80^{\circ} \mathrm{C}$ the temperature flow can be stabilized.

6. LOW TEMPERATURE FLASH MEMORY APPLICATION: This research discusses about the voltage to capacitance where the storage capacity is significantly increased with the hysteresis curves and capacitance-voltage $(\mathrm{C}-\mathrm{V})$. this relates to $\mathrm{HfO} 2$ based charge trap flash memory.

Observation: The samples are studied and the $\mathrm{SiO} 2$ film is thermally grown at $900{ }^{\circ} \mathrm{C}$ and with the atomic layer deposition process and the substrate is varied at different temperatures randomly with the $700{ }^{\circ} \mathrm{C}$ the structure is then annealed. With the $\mathrm{Al}$ gate the electrode is patterned with the spectroscope and it is measured at $100 \mathrm{k} \mathrm{Hz}$ to extract the flat band voltage. The TEM images varies between $150{ }^{\circ} \mathrm{C}$ and $350{ }^{\circ} \mathrm{C}$ where the MHOS devices a distinct interface which can be observed between the $\mathrm{SiO} 2$ and $\mathrm{HfO} 2$. The device is fabricated using the specifications.

Outcome: This work relates to the thin film memory device which is manufactured through the Atomic Layer Deposition the capacitance-voltage, substrate temperature and the 
parameters are observed. It is a significant that the storage capacity can be improvised by using this technique.

7. GRAPHENE QUANTUM DOTS: This study relates with the properties of the graphene in memory applications. this advancement has increased the biologically inert properties which is attracted by the academic and industry. This is a hydrothermal method is summarized. As the summary represents the doping of the energy in the Graphene is highly useful in biological imaging, batteries, memory devices, solar cells .etc.,

Method: The graphene can be manufactured using two methods namely top down and bottom up approach. Which is directing the bulk carbon materials into the nano sale with the lithography technique. It uses the poly cyclic hydrocarbons which are molecule in the graphene dots and external energy is provided for fabrication.

Synthesis: using liquid exfoliation method the preparation of graphene is from the graphite powder where it is performed using strong oxidizing agents. The synthesis of graphene quantum dots usually requires the acid which cuts the graphene. Bulk carbon materials will be obtained that has to be filtered in layers and graphene is formed. Usually coal or graphite is used to obtain the graphene.

Outcome: This review results with manufacturing of graphene with various materials fabrication of the graphene with memory devices has unique properties and the it is achieved that the layered graphene has an higher potential when compared with other materials such as lithium ion which is mostly used in day to day applications. this review discusses about the possibilities, developments and fabrication methods of the graphene.

8. NANO DOTS ON REDUCED GRAPHENE: This study relates to the Au nano dots on graphene oxide in this process the rapid reduction in the metallic nanodots on the surface is done. This has broader visible light absorption, lower generation in electrons, fast electron transfer. It has an potential application in the memory and storage applications.

Observation: Nanodot heterostructures are 2D graphene with metal oxides and it has an potential application in the memory and energy consumption. The nanostructures which is used are MoS2, TiO2, CdS, Mn doped $\alpha$-Fe2O3, $\alpha$-Fe2O3/3D graphene, $\alpha$-Fe2O3/reduced graphene oxide and Au-supported with a low bandgap to absorb the photons. $\alpha-\mathrm{Fe} 2 \mathrm{O} 3$ is used with the carbon nanotubes and reduced graphene with the nanosheets.

Outcome: this review results with a green synthesis method of $\alpha$-Fe2O3 under sunlight this generates the $\mathrm{Au} \mathrm{3+}$ ions. it has large specific surface area, thermal conductivity, light transmission and good mechanical strength. This can be an active material in production of the memory devices, energy storage and conductors

9. NANO STRUCTURED HYDRODIXE SYNTHESIS: This work relates to the synthesis of iron oxides with various methods such as top-bottom method, chemical method, precipitation. iron oxides in the nanoscale has an great potential in various industry related applications one among is memory device manufacturing.

Synthesis: This iron oxides is an active research topic it has an unique features like concentration, temperature, mixing and oxidation. This depends on the desired shape and size. the synthesis follows slow, continuous and surface growth. The electrochemical synthesis of nano sized particles is non-aqueous medium in which $20 \mathrm{~nm}$ in the aqueous medium is taken and the stainless steel is used as a cathode and anode. 3-8 nm of maghemite

particles can be re-used in an aqueous solution in the cationic surface. This can be used in manufacturing of nano dots, nano wires.

Outcome: the iron oxide nano particles can be used in various industries such as memory devices, charge storage, medical applications, chemical industries and water purification. The size has been reduced when compared to other materials. 


\section{Conclusion}

1. When compared to the above materials and processes the production using $\alpha$ $\mathrm{Fe} 2 \mathrm{O} 3$ and $\mathrm{Au}$ nanodots can improve the performance, speed, and size can be reduced.

2. The Nano particles can be synthesized by using precipitation, sol-gel, hydrothermal, dry vapor deposition, microemulsion, electro deposition.

3. The above mentioned comparisons are reviewed with the $\alpha-\mathrm{Fe} 2 \mathrm{O} 3$ green synthesis which can be environment friendly and which is bio degradable and can be used in various medical applications.

4. Green synthesis of $\alpha-\mathrm{Fe} 2 \mathrm{O} 3$ with $\mathrm{Au}$ can be most efficient when compared to other materials which are available today.

5. When compared to graphene the green synthesis is more viable as the cost of extraction of graphene is higher and difficult.

In conclusion this review has provided the developments, fabrication methods, it can be helpful in the green synthesis and it can be $30 \%$ more efficient when compared to the present methodologies as the process used in manufacturing of the $\alpha$ - $\mathrm{Fe} 2 \mathrm{O} 3$ with Au nano dots can be a viable method.

\section{References}

[1] S.Y. Lim, W. Shen, Z. Gao, Carbon quantum dots and their applications. Chem. Soc. Rev. 44, 362 381 (2015).

[2] D. K. Bora, A. Braun, and E. C. Constable,Energy Environ. Sci.6, 407(2013).

[3] G. Bharath, et al, Sunlight-Induced photochemical synthesis of Au nanodots on $\alpha$ Fe2O3@Reduced graphene oxide nanocomposite and their enhanced heterogeneous catalytic properties

[4] P. Tian et al, Graphene Quantum dots from chemistry to applications. Revise 221-258 (2018).

[5] G. Haider, et al, Electrical-polarization-induced ultrahigh responsivity of photo- detectors based on graphene alloys and graphene quantum dots, Advanced. Functional Mater. 4 (2016).

[6] P.C.Wu, et al. Efficient two photon luminescence for cellular imaging using biocompatible nitrogen doped graphene quantum dots conjugated with polymers, Nanoscale 1 (2018)

[7] H. Chen, Z. Wang, S. Zong, P. Chen, D. Zhu, L. Wu, Y. Cui, A graphene quantum dot- based FRET system for nuclear-targeted and real-time monitoring of drug delivery, Nanoscale 7 (2015)

[8] A. Dhand, S. Suresh, A. Jain, O.N. Varadan, M.A.K. Kerawalla, P. Goswami,Advances in materials for fuel cell technologies-a review, Int. J. Res. Appl. Sci.Eng. Technol. 5 (2017) 1672e1682.

[9] Q. Liu, Y. Yin, N. Hao, J. Qian, H. Mao, K. Wang, Self-templating synthesis ofnitrogen doped graphene quantum dots/3D bismuth oxyiodine hybrid hol-low microspheres with improved visiblelight excited photocurrent gener-ation: simultaneous electron transfer acceleration and bandgap narrowing,J. Alloy. Comp. 729 (2017) 27e37

[10] Yating Zhang, et al. Preparation of coal based graphene quantum dots $/ \alpha-\mathrm{Fe} 2 \mathrm{O} 3$ nano alloy composites and their li-ion storage prop. Rev 646-652 (2019)

[11] Wang Y, Roller J, Maric R. Novelflame synthesis of nanostructured $\alpha$-Fe2O3elec-trode as highperformance anode for lithium ion batteries. J Power Sources2018;378:511-5.

[12] Zenhan zhang, et al. Memory materials and devices: From concept to application. SEP 2019

[13] Chand U, Huang K-C, Huang C-Y, Tseng T-Y. Mechanism ofnonlinear switching in HfO2-based crossbar RRAM withinserting large bandgap tunneling barrier layer.IEEE TransElectron Devices. 2015;62:3665-3670

[14] Woo J, Lee W, Park S, et al. Multi-layer tunnel barrier(Ta2O5/TaOx/TiO2) engineering for bipolar RRAM selectorapplications. Paper presented at: IEEE Symposium on VLSIT,2013:T168-T169 
[15] Yu S, Wu Y, Jeyasingh R, Kuzum D, H-SP W. An electronicsynapse device based on metal oxide resistive switching mem-ory for neuromorphic computation.IEEE Trans ElectronDevices.2011;58:2729-2737

[16] Waser R, Dittmann R, Staikov G, Szot K. Redox-based resistive switching memories- nano ionic mechanism, prospect and challenges.Advanced Mater. 2010;21:2632-2663

[17] Yu S, Chen H-Y, Gao B, Kang J, H-SP W. HfOx-based verticalresistive switching random access memory suitable for bit-cost-effective three-dimensional cross-point architecture.ACSNano. 2013;7:2320-2325

[18] Tappertzhofen S, Waser R, Valov I. Impact of the counter-electrode material on redox processes in resistive switchingmemories.ChemElectroChem. 2014;1:1287-1292.

[19] Splendiani A, Sun L, Zhang Y, et al. Emerging photo-luminescence in monolayer MoS2.Nano Lett. 2010;10:1271-1275

[20] Liu C, Yan X, Song X, Ding S, Zhang DW, Zhou P. A semi-floating gate memory based on van der Waals hetero-structures for quasi-non-volatile applications.NatNanotechnol. 2018;13:404-410

[21] Fossum ER. CMOS image sensor aloy: electronic camera on a chip with memory dopant. Trans Electron Devices. 1997;44:1689-1698.

[22] Wu F, Si S, Cao P, et al. Interface engineering via MoS2inser-tion layer for improving resistive switching of conductive-bridging random access memory. Adv Electron Mater.2019;5:1800747.

[23] Park, S.G.; Köpe, B.M.; Nishi, Y. Theoretical study of the resistance switching mechanism in rutile TiO2-xforReRAM: The role of oxygen vacancies and hydrogen impurities. In Proceedings of the 2011 Symposium onVLSI Technology - Digest of Technical Papers, Honolulu, HI, USA, 14-16 June 2011.

[24] Ho, C.H.; Hsu, C.-L.; Chen, C.-C.; Liu, J.-T.; Wu, C.-S.; Huang, C.-C.; Hu, C.; Yang, F.-L. 9nm half-pitchfunctional resistive memory cell with $<1 \mu \mathrm{A}$ programming current using thermally oxidized sub-stoichiometricWOx film. In Proceedings of the 2010 International Electron Devices Meeting, San Francisco, CA, USA, 6-8December 2010; pp. 436-439

[25] Kozicki, M.N.; West, W.C. "Programmable Metallization Cell Structure and Method of Making". US PatentUS5761115A, 2 June 1998.

[26] G. González-Cordero, J.B. Roldán, F. Jiménez-Molinos, SPICE simulation of RRAMcircuits. Acompact modeling perspective. 2017

[27] X. Guan, S. Yu, and H.S.P.Wong, "A SPICE Compact Model of Metal Oxide Resistive Switching Memory with Variations", IEEE Elec. Dev. Lett., vol. 33, pp. 1405-1407, 2012.

[28] P. Y. Chen and S. Yu, "Compact Modeling of RRAM Devices and Its Applications in 1T1R and 1S1R Array Design",in IEEE Transactions on Electron Devices, vol. 62, no. 12, pp. 4022-4028, Dec. 2015 .

[29] S. Long, L. Perniola, C. Cagli, J. Buckley, X. Lian, E. Miranda, F. Pan, M. Liu and J. Suñé. "Voltage and power-controlled regimes in the progressive unipolar RESET transition of HfO2based RRAM",Scientific reports, 3, 2013.

[30] International Roadmap for Devices and Systems. "BeyondCMOS,"2017.

[31] N. Raghavan, R. Degraeve, A. Fantini, L. Goux, D. J.Wouters, G. Groeseneken, and M. Jurczak, "Stochasticvariability of vacancy filament configuration in ultra-thindielectric RRAM and its impact on OFF-state reliability,"in2013 IEEE International Electron Devices Meeting,Wash-ington, DC, Dec.2013, pp. 21.1.1-4.

[32] Victor Koledov, et al. 3D Nano-Manipulation and Nano-assembling by the smallest and the fastest shape memory alloy nano-tools. 2019

[33] V.S.Afonina, D.I. Zakharov, A.V.Irzhak, V.V.Koledov, P.V.Lega, A.V.Mashirov, et al. "Micromechanical device, the manufacturing method and system of manipulation of micro- and nanoobjects." Russian Patent No 2458002, 15.08.2010.

[34] Stefan Slesazeck, Nanoscale resistive switching memorydevices: a review. 2019.

[35] Changhyuck Sung, Jeonghwan Song, Donguk Lee, Seokjae Lim, Myounghun Kwakand Hyunsang Hwang, Ultra-thin $(<10 \mathrm{~nm})$ Dual-oxide (Al2O3/TiO2) Hybrid Device (Memory/Selector) with Extremely Low Ioff $(<1 \mathrm{nA})$ and Ireset $(<1 \mathrm{nA})$ for 3D Storage Class Memory. 2019. 